\title{
RNA-Seq Reveals the Effect of Ethylene on the Volatile Organic Components (VOCs) of Cavendish Banana at Different Post-harvesting Stages
}

\author{
Tongxin Dou ${ }^{1,2,3}$ (D) Chunhua Hu ${ }^{1,2,3}$. Shujing Zhao ${ }^{1}$ - Huijun Gao ${ }^{1,2,3}$. Weidi $\mathrm{He}^{1,2,3}$. Guiming Deng ${ }^{1,2,3}$. \\ Ou Sheng ${ }^{1,2,3} \cdot$ Fangcheng $\mathrm{Bi}^{1,2,3} \cdot$ Qiaosong Yang ${ }^{1,2,3} \cdot$ Chunyu $\mathrm{Li}^{1,2,3} \cdot$ Ganjun $\mathrm{Yi}^{1,2,3} \cdot$ Tao Dong ${ }^{1,2,3}$
}

Received: 3 July 2020 / Accepted: 18 February 2021 / Published online: 29 September 2021

(c) The Author(s) 2021

\begin{abstract}
Aroma serves as one of the decisive factors influencing the value of banana commodities. Most of characteristic volatile organic components (VOCs) are formed during post-harvesting. However, the changing of VOCs of banana at different post-harvesting stages remain ambiguous. In this study, the VOCs of Cavendish banana for the four typical post-harvesting stages (green stage/half of yellow stage/yellow ripening stage/over ripening stage) are clarified using headspace solid phase micro-extraction (HS-SPME), combined with gas chromatography-mass spectrometry (GC-MS). The results inferred that the relative content of branched-chain esters such as acetate and butyrate, which form the main contributors of aroma in bananas, is higher in the T2 and T3 stages. Further, RNA-Seq technology was employed to clarify the formation mechanism of banana aroma in the post-harvesting stage. The MaTGL4 gene of the linoleic acid metabolism pathway and the MaBCAT3 and MaBCAT5 genes of the valine, leucine and isoleucine degradation pathway in banana suggest the expression is active late in the ripening stage, and the upregulated expression of these genes is analogous to the formation of aroma components such as branched-chain esters and hexenal. The above results not only provide baseline data on the differences in physical and chemical properties of VOCs in various post-harvesting stages of banana production, but also provide theoretical guidance facilitating the subsequent improvement of the commercial value of bananas through genetic improvement.
\end{abstract}

Keywords Banana $\cdot$ Candidate genes $\cdot$ Fruit ripening $\cdot$ Transcriptome $\cdot$ Volatile aroma components

\section{Abbreviations}

VOCs Volatile organic components

HS-SPME Headspace solid phase micro-extraction

GC-MS Gas chromatography-mass

Handling Editor: Fabrizio Costa.

Tongxin Dou and Chunhua Hu have contributed equally to this work.

Tao Dong

taod2004@163.com

1 Institute of Fruit Tree Research, Guangdong Academy of Agricultural Sciences, No. 80 Dafeng 2nd Street, Tianhe District, Guangzhou 510640, People's Republic of China

2 Key Laboratory of South Subtropical Fruit Biology and Genetic Resource Utilization, Ministry of Agriculture, Guangzhou 510640, People's Republic of China

3 Key Laboratory of Tropical and Subtropical Fruit Tree Research, Guangzhou 510640, Guangdong Province, People's Republic of China
BCAAs Branched-chain amino acids

DEG Differentially expressed genes

\section{Introduction}

During the course of growth and development, plants tend to produce volatile organic components (VOCs) like terpenes. Typically found to be present in the edible part of plants like the plants' rhizome, these volatile substances exhibit vital organic sensory characteristics responsible for the formation of flavor, which affects the preferences of consumers (Frérot et al. 2017; Santos et al. 2017; Chen et al. 2018). VOCs predominantly include esters, alcohols, phenols, ketones, aldehydes, ethers, and terpenes (Yvon and Rijnen 2001; Ardö 2005; Itay et al. 2010).

Banana (Musa spp) is not just considered one of the world's major fruit crops, but also serves as a staple food item for more than 400 million people in more than 130 countries such as Africa, America, and Asia, and enjoys 
pivotal economic stature (Rustagi et al. 2015; Wei et al. 2017; Li et al. 2020). Aroma is one of the most appreciated characteristics of any fruit and is seen as a profound determining factor for its acceptability by consumers (Selli et al. 2012; Dou et al. 2020). As a vital economic fruit, banana is rich in VOCs, which include esters, alcohols, phenols, ketones, aldehydes, ethers, and terpenes (Boudhrioua et al. 2003). VOCs are highly accountable for the aromatic flavor of banana. Aroma constituents of banana (Facundo et al. 2012; Dong et al. 2014; Bugaud and Alter 2016; Saha et al. 2018) have been extensively studied. Approximately 250 volatile compounds have been detected in banana at different stages of ripeness and process (Jordan et al. 2001; Pino and Febles 2013). Generally, the major volatile compounds are classified as esters, alcohols, and ketones. Furthermore, several reports suggest that acetates (especially isoamyl acetate and isobutyl acetate) and butyrate are highly influential for the development of aroma of a fully ripe banana fruit (Marriott and Palmer 1980; Hadi et al. 2013). Nogueira et al. (2003) investigated the composition of volatiles found in banana cultivars (Dwarf Cavendish, Giant Cavendish, Robusta and Williams) from Madeira Island. It was deduced that the ester $(57.2-89.8 \mathrm{mg} / \mathrm{kg})$ and alcoholic fractions $(19.0-47.7 \mathrm{mg} / \mathrm{kg})$ of the volatiles observed appeared to play a decisive role in the aroma characteristics of banana fruit. Experiments using GC-olfactometry (Gas Chromatography Olfactometry) strongly advocated for 3-methylbutylacetate, 3-methyl-butyl butanoate, and 3-methylbutyl3-methyl butanoate to be the key components responsible for banana's fruity odor (Facundo et al. 2012).

Ripening tends to be the amalgamation of processes that initiate at the latter stages of growth and proceed up to the early stages of senescence, resulting in the genesis of characteristic properties of the ripe fruit-like flavor, taste, texture, color. Ripeness being the determining parameter for substrate availability in the plant system, in turn makes biogenesis of fruit aroma evidently dependent on the ripeness of the material. At varied stages of ripening, Zhu et al. (2010) investigated the transformations of the volatile components of banana fruit, simultaneously exploring the relationships between volatile production and other physiological events occurring during fruit ripening. Moreover, it was reported that changes in the production of total volatile compounds by banana fruit during the process of storage were closely affiliated to the event of fruit ripening and the ripeningassociated parameters, such as fruit ethylene production, respiration rate, firmness, color, soluble solids content, and starch content (Zhu et al. 2010). Also, some volatiles come across as particularly good indicators of the ripening stage of bananas, such as hexanal and (E)-2-hexenal, as well as isobutyl acetate and isopentyl acetate, which are characteristic of ripe and ripening bananas (Mayr et al. 2003).
It is evidently known that both fatty acid and amino acid pathways can produce aldehyde, followed by reduction to alcohol by alcohol dehydrogenase (ADH) (Speirs et al. 1998), and finally generate esters catalyzed by alcohol acyltransferase (AAT) (Beekwilder et al. 2004; Souleyre et al. 2014). Studies have proven that leucine, isoleucine, valine, and alanine are the major biosynthetic precursors of branched-chain esters in banana, strawberry and several other fruits that are closely related to the formation of esters in fruits (Wyllie and Fellman 2000; Pérez et al. 2002). Considering the primary aroma of several fruits (Pérez et al. 2002), these compounds either act as major contributors or behave as determining factors in some cases. Due to the development of 'Omics technology' (universal detectors of complete genetic or molecular profile of any organism, classified into genomics, transcriptomics, etc.,) in recent years, several genes closely associated with the synthesizing of banana volatile substances have been isolated and identified. For example, the genes encoding aroma synthesis in banana fruit have been partially identified, including O-methyltransferases 1 (OMT1), branched-chain amino acid transaminase 1 (BCAT1), pyruvate dehydrogenase complex $(P D C)$, basic leucine zipper 4 ( $b Z I P 4)$, basic leucine zipper 5 (bZIP5), MaADH, and BanAAT (Yang et al. 2011; Guo et al. 2018). Some studies conducted by Asif et al. (2014) suggested that the expression of $M a A D H$ demonstrated more than twofold upregulation in the case of a ripe fruit as compared to MaOMT, MaBCATl, and MaAAT genes and some other unripe fruit. As mentioned above, previous studies have described the major contributors of banana volatile aroma. This paper undertakes a preliminary exploration at the genetic level to further divulge in comprehending the mechanism of banana aroma substances formation. However, the changing rules and mechanisms of banana VOCs at different post-harvesting stages remain vague and cryptic.

In this study, firstly, HS-SPME coupled with GC-MS technology was employed to evaluate the changing rules of banana VOCs in different post-harvesting stages. Geared towards clarifying the formation mechanism of banana VOCs in various post-harvesting stages, conduction of RNA-Seq sequencing analysis for four typical post-harvesting stages of banana fruits was undertaken. Several studies advocate that the relative content of branched-chain esters and branched-chain alcohols in the late ripening stage of banana fruits shoots up significantly, and three certain genes related to lipid and amino acid metabolism are actively expressed in the late banana ripening stage, noticeably predicting their impending correlation with the formation of aroma substances in the later stages of banana ripening. These findings serve as a robust stand on the basis of which further in-depth analysis of the metabolic mechanism of aromatic substances during banana ripening at the molecular level could be undertaken. 


\section{Materials and Methods}

\section{Plant Materials and Treatment}

The cultivar of mature, green Cavendish banana fruit (Musa acuminate, AAA group, cv. Cavendish) at the $75-80 \%$ plump stage were harvested from the plantation $\left(23^{\circ} 75^{\prime} \mathrm{N}\right.$, $114^{\circ} 25^{\prime}$ E) of Institute of Fruit Tree Research, Guangdong Academy of Agricultural Sciences. To rule out the impact of different harvest seasons on fruit aroma, banana bunches were harvested at the same time and under the same physiological maturity stage (caliber $33 \pm 1 \mathrm{~mm}$, measured in the middle finger of outer whorl of the second hand from the distal end of the bunch). The second hand from the proximal end of each bunch was selected (around $60 \mathrm{~kg}$ of banana), then dipped in $1.0 \mu \mathrm{l} / \mathrm{L}$ ethephon for $1 \mathrm{~min}$. The fruit was naturally dried and then stored in a polyethylene film bag at $18{ }^{\circ} \mathrm{C}$ for $36 \mathrm{~h}$. Post that, the bag was opened but continued to be placed at $18{ }^{\circ} \mathrm{C}$ until the banana fruit completed the whole post-harvesting process. According to the peel color measurement results and phenotype changes, it is determined that the 1 st day of post-harvesting without ethephon treatment is the green stage (CK), the 3rd day after ethephon treatment is half of yellow stage (T1), the 6th day after ethephon treatment is yellow ripening stage (T2), and the 9th day after ethephon treatment is over ripening stage (T3). The fruits were peeled, and their pulp was sliced, frozen in liquid nitrogen and stored at $-80^{\circ} \mathrm{C}$ for subsequent transcriptome analysis. Three independent measurements were conducted on randomly selected fruits as replicates for each ripening stages.

\section{Color of Banana Peels}

The color of banana fruit was analyzed and measured according to the method we reported previously (Dou et al. 2020). The colorimetric coordinates of fresh banana peels were measured using a Minolta Chroma Meter CR 400 (color space CIE L*, $\mathrm{a}^{*}, \mathrm{~b}^{*}$ and $\mathrm{h}^{*}$ ). To avoid experimental errors, three banana fruits were randomly tested at each time point, and three fixed parts were tested for each fruit.

\section{Volatiles Analysis}

The VOCs of bananas were determined according to the method previously reported in this study (Dou et al. 2020). The 50/30 $\mu$ m Divinylbenzene/Carboxen/Polydimethylsiloxane (DVB/CAR/PDMS) fibers extraction head manufactured by Supelco, Bellefonte, Pa., U.S.A was used for the test. The extraction head was aged at $250^{\circ} \mathrm{C}$ for $2 \mathrm{~h} .20 \mathrm{~g}$ of homogenate was sealed in a $50 \mathrm{ml}$ headspace vial. Solid-Phase Micro extraction (SPME) was carried out for $30 \mathrm{~min}$ and desorbed at $220^{\circ} \mathrm{C}$ for $3 \mathrm{~min}$. Samples were analyzed using a Finnigan Trace GC-MS instrument. The GC-MS conditions were: DB-1 column (length $30 \mathrm{~m}$, inner diameter $0.25 \mathrm{~mm}$, liquid film thickness $0.1 \mu \mathrm{m}$ ), column temperature $40^{\circ} \mathrm{C}$ for $1 \mathrm{~min}$, then increased by $2{ }^{\circ} \mathrm{C} / \mathrm{min}$ to $60^{\circ} \mathrm{C}$ (for $2 \mathrm{~min}$ ), and further increased by $10^{\circ} \mathrm{C} / \mathrm{min}$ to $180^{\circ} \mathrm{C}$ (for $5 \mathrm{~min}$ ). The inlet temperature was $220^{\circ} \mathrm{C}$. Carrier gas $\mathrm{He}(99.99 \%)$ was provided at a flow rate of $1.0 \mathrm{~mL} / \mathrm{min}$. The EI ion source has a temperature of $170{ }^{\circ} \mathrm{C}$, an electron energy of $70 \mathrm{eV}$, and a photomultiplier tube voltage of $350 \mathrm{~V}$ was employed. The mass scan range was 35-335 amu. The total ion chromatogram of the collected aroma substances was determined using the NIST 11 and Wiley mass spectral libraries to determine the chemical composition of the aroma components. The relative content of each component was obtained using the peak area normalization method for quantification.

\section{RNA Extraction and RNA-Sequencing}

Total RNA derived from banana pulp was isolated using a plant RNA extraction kit (Code No. 9767, TaKaRa, Dalian, China), and first strand cDNA was synthesized from $1 \mu \mathrm{g}$ of total RNA using a PrimeScript RT Reagent Kit, according to the manufacturer's instructions (TaKaRa). Triplicates were made at each time point, and each biological replicate randomly selected a mixed sample of three fruit pulps. RNA preparation, library construction, and sequencing on the BGISEQ-500 platform of PE 100 with 30 million reads per sample was performed at BGI (http://www.genomics. org.cn, BGI, Shenzhen, China). Gene expression levels were quantified using the RSEM software package (Li and Dewey 2011). The DEGseq method was utilized to screen differentially expressed genes (DEGs) between groups as previously described in this study (Huang et al. 2018). Gene Ontology (GO) and pathway annotation and enrichment analyses were based on the GO Database (http://www.geneontology.org/) and the KEGG pathway database (http://www.genome. jp/ $\mathrm{kegg} /$ ), respectively. Statistical analysis was performed and DEGs were selected with the criteria of a fold change $\geq 2$ and adjusted $p$ value $\leq 0.001$, as described previously (Huang et al. 2018).

\section{Quantitative Real-Time (RT-qPCR) PCR Analysis}

To further validate the reliability of this transcriptomic data, total RNA derived from banana pulp at four different time points was isolated using a plant RNA extraction kit (Code No. 9767, TaKaRa, Dalian, China), and the first strand cDNA was synthesized from $1 \mu \mathrm{g}$ of total RNA using a PrimeScript RT Reagent Kit, according to the manufacturer's instructions (TaKaRa). The resulting cDNAs were then employed as templates for qRT-PCR. 8 genes were 
assayed by RT-qPCR with specific primers as shown in File S3. The PCR reaction consisted of $10 \mu \mathrm{L}$ of $2 \times$ SYBR Green PCR Master Mix (TaKaRa), $200 \mathrm{nM}$ primers, and $2 \mu \mathrm{L}$ of 1:40-diluted template cDNA in a total volume of $20 \mu \mathrm{L}$. The RT-qPCR program included an initial denaturation step at $95{ }^{\circ} \mathrm{C}$ for $10 \mathrm{~s}$, followed by 40 cycles of $5 \mathrm{~s}$ at $95^{\circ} \mathrm{C}$, and annealing and extension at $60{ }^{\circ} \mathrm{C}$ for $20 \mathrm{~s}$. The MaACT1 gene (File S3) was selected as a reference gene base on our previous report (Dou et al. 2016). The relative expression levels of the amplified products were calculated based on the comparative threshold cycle method (Li and Dewey 2011). Transcript abundance of the 8 candidate genes was normalized against the reference gene, and the three technical replicates were performed for each gene.

\section{Results and Discussion}

\section{Determination of Typical Post-harvesting Stage of Banana Fruit}

The banana peel gradually turned yellow, and subsequently on the 9th day of storage, the banana peel demonstrated small sesame spots (Fig. 1a). Afterwards, the fruit rotted and lost its commercial value. In order to determine the time point of sampling at different post-harvesting stages of bananas, we measured the color of banana peels, as shown in Fig. 1b. With the extension of storage time, the red saturation $\mathrm{a}^{*}$ value proclaimed a recent upward trend, but $\mathrm{h}^{*}$ portrayed the gradual decrease trend, inferring that the banana fruit gradually completed the post-harvesting process; both $L^{*}$ and $b^{*}$ initially showed an elevating trend but then led to a decline, and both reached the maximum value on the 6th day of storage, indicating that the banana fruit had completed the post-mature date since the period after 6 days is the over-mature stage.

Previous studies demonstrate that volatile components of banana fruits are affected by genotype (Aurore et al. 2011; Pontes et al. 2012; Bugaud and Alter 2016), cultural practice (Brat et al. 2004; Dong et al. 2014), stages of ripening (Zhu et al. 2018), processing conditions (Wang et al. 2007), harvest season (Shivashankara et al. 2017; Dou et al. 2020) and storage conditions (Boudhrioua et al. 2003), along with several other factors. These factors tend to regulate the quantitative and qualitative composition of the volatile profile, which in most cases determines the character and perception of quality of the fruits (Brat et al. 2004). Therefore, exploring the changing rules and mechanisms of aroma components of
Fig. 1 The color change of banana fruit at different postharvesting stages. a $1.0 \mu \mathrm{l} / \mathrm{L}$ ethephon for $1 \mathrm{~min}$. The fruit was naturally dried and stored in a polyethylene film bag for $36 \mathrm{~h}$ at $18^{\circ} \mathrm{C}$, after that, the bag was opened but continued to be placed at $18{ }^{\circ} \mathrm{C}$. The banana fruit completes the whole postharvesting process, of which $\mathrm{CK}, \mathrm{T} 1, \mathrm{~T} 2$, and $\mathrm{T} 3$ are four typical post-harvesting stages. $\mathbf{b}$ Color changes of banana peels at different post-harvesting stages (color space CIE L *, a *, $\mathrm{b} *$ and $\mathrm{h} *)$ $\mathbf{a}$

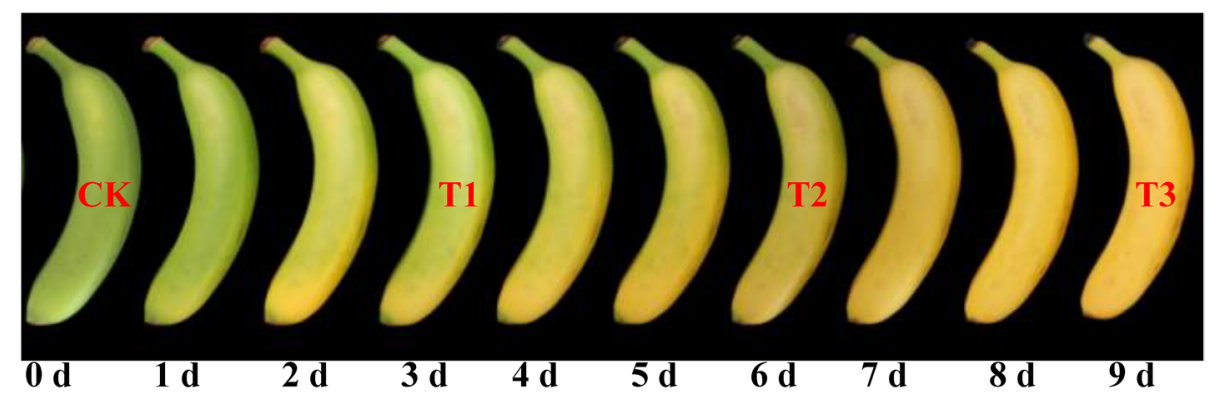

b

banana fruits at different post-harvesting stages proves to be an extensively vital step. Whereas, determining the typical post-harvesting stage is the crucial link of this study.

\section{Variation of Banana Volatile Components at Different Post-harvesting Stages}

Combining dynamic headspace solid-phase micro-extraction with one-dimensional gas chromatography-mass spectrometry (GC-MS) serves as a powerful tool to differentiate banana cultivars based on their volatile metabolite profile (Dou et al. 2020). To further explore the changing rules of the aroma components of banana fruits at various post-harvesting stages, the above four post-harvesting periods were selected, HP-SPME and GC-MS were adopted to analyze the volatile compounds, and the compounds were identified based on the MS data. As shown in Table 1, in the post-harvesting stage of bananas, a total of 87 volatile components were detected, which mainly include alcohol, ester, aldehyde, acid, hydrocarbon, ketone and several other compounds.

The main aroma components of banana are: Acetic acid; 2-methylpropyl ester/1-Butanol; 3-methyl-; acetate/Butanoic acid; 3-methylbutyl ester/Butanoic acid; 3-methyl-; and 3-methylbutyl ester, which are mainly formed by fatty acid metabolism of ester acyl-CoA and the corresponding amino acid metabolite isobutanol or isoamyl alcohol (Jordan et al. 2001; Bugaud and Alter 2016). As shown in Table 1, the ester components in this study were mainly detected in the $\mathrm{T} 2$ and $\mathrm{T} 3$ stages, with relative content of $8.45 \%$ and $26.60 \%$, respectively. The ester content detected in the CK and $\mathrm{T} 1$ stages were only about $0.14 \%$ and $0.47 \%$, respectively. Also, the esters detected in the T2 and T3 stages mainly turn out to be acetate $(2.67 \%, 18.03 \%)$ and butyrate $(4.3 \%$ and $5.72 \%)$. They are predominantly the main aroma substances of banana flavor, while the acetate can emit an attractively strong scent (Wyllie and Fellman 2000) even when present in low concentration. The biosynthetic pathway for their formation in ripening climacteric fruits, such as apples, bananas, apricots, is well-established. Several investigations demonstrate that the volatile esters that are produced by fruits are not only determined by AAT specificity but also by the substrate availability, which is highly dependent on the ripening stage of the fruit (Beekwilder et al. 2004).

The relative content of alcohol compounds in the four different post-harvesting stages is $10.75 \%, 12.60 \%, 9.13 \%$, and $18.94 \%$, respectively, and the composition of the alcohol component tends to be significantly distinct in each stage, among which the alcohol detected in the CK and T1 stages is mainly sitosterol, while the alcohol traces in T2 and T3 phases are mainly 1-Butanol; 3-methyl-, 1-Propanol; 2-methyl-, (S)-(+)-2-Pentanol and 1,2-Propanediol; 3-methoxy- and other branched short fatty alcohol (Table 1). However, alcohols remain less crucial as flavor molecules due to their high odor thresholds as compared to their aldehyde homologues (Marriott and Palmer 1980). Branchedchain fatty alcohols seem to be closely related to amino acid catabolism (Xu et al. 2017).

Both alcohols and minor carbonyl compounds are referred to as green and woody notes. (E) and (Z)-hept-4-en-2-one have fatty-green and sweet notes (Miranda et al. 2001; Pino et al. 2017). As shown in Table 1, the detected acids are mainly medium and long-chain fatty acids. The fatty acids detected in the $\mathrm{CK}$ and $\mathrm{T} 1$ phases are relatively high, at $58.20 \%$ and $63.46 \%$, respectively; the contents of the $\mathrm{T} 2$ and $\mathrm{T} 3$ phases are relatively low, at $37.22 \%$ and $17.82 \%$, respectively. The main acid component is identified as n-Hexadecanoic acid, whose ratios in CK/T1/T2/T3 stage are 29.22\%, $24.03 \%, 22.00 \%$, and $9.77 \%$, respectively. The detected aldehydes are mainly Hexanal and 2-Hexenal, (E)-, whose relative content is higher in the T2 and T3 stages, but present in minute traces in the $\mathrm{CK}$ and $\mathrm{T} 1$ stages. The formation of Hexanal and 2-Hexenal, (E)- comes from the degradation of fatty acids (Yvon and Rijnen 2001; Ardö 2005). The detected hydrocarbons are mainly straight-chain alkanes or olefins. There are fewer types of hydrocarbon components detected in the T2 and T3 stages, and the content of the hydrocarbon components in the T3 stage is at least $1.82 \%$. In addition, some ethers, amides, and benzene rings in different postharvesting stages of bananas were detected as well.

\section{Differential Expression of Genes During Post-harvesting of Bananas}

The post-harvesting process of banana fruits marks the involvement of many genetic differences (Asif et al. 2014). In order to explore the formation mechanism of banana aroma substances at different post-harvesting stages and to screen and analyze candidate genes involved in the formation of banana aroma substances, we used CK as the comparison group for reference and $\mathrm{T} 1, \mathrm{~T} 2$, and $\mathrm{T} 3$ stages as different post-harvesting stages of bananas. The RNAseq high-throughput sequencing was performed separately. The sequencing instrument was: illumina Hiseq 2500, and the sequencing strategy was double-end reads $150 \mathrm{bp}$, that is, PE 150 sequencing. The DESeq2 software was utilized to analyze the difference between the two groups. The screening conditions for the differential genes were: FDR value less than 0.05 and difference multiple $\geq 2$. As shown in Fig. 2, compared to CK, there are 4277 genes upregulated and 8,412 genes downregulated in T1 phase; 4587 genes upregulated and 8765 genes downregulated in T2 phase; 4292 genes upregulated and 8923 genes downregulated in T3 phase; 9630 genes have differential expression changes 
Table 1 Volatiles compounds content in M. acuminata fruit harvest at different ripening stage

\begin{tabular}{|c|c|c|c|c|c|}
\hline \multirow[t]{2}{*}{ Retention time } & \multirow[t]{2}{*}{ Volatile compounds } & \multicolumn{4}{|c|}{ Relative content $(\%)$} \\
\hline & & CK & $\mathrm{T} 1$ & $\mathrm{~T} 2$ & T3 \\
\hline \multicolumn{6}{|l|}{ Alcohols } \\
\hline 2.05 & 1-Propanol; 2-methyl- & - & - & - & 4.91 \\
\hline 2.60 & $(\mathrm{~S})-(+)-2$-Pentanol & - & - & 1.39 & 1.92 \\
\hline 2.99 & 1-Butanol; 3-methyl- & - & - & 3.53 & 6.85 \\
\hline 4.01 & 1,2-Propanediol; 3-methoxy- & 1.18 & 0.75 & 2.43 & 1.37 \\
\hline 5.00 & 1-Hexanol & 0.08 & 0.41 & 0.29 & 0.21 \\
\hline 8.83 & 4-Nonanol & 1.06 & 1.18 & 1.50 & 1.52 \\
\hline 10.80 & 3-Pentanol; 2,4-dimethyl- & - & - & - & 0.26 \\
\hline 12.94 & 1-Heptanol; 2-propyl- & 0.54 & - & - & - \\
\hline 22.10 & n-Tetracosanol-1 & 0.53 & 0.80 & - & - \\
\hline 25.45 & 1-Heptacosanol & 0.18 & 0.29 & - & - \\
\hline 25.84 & .gamma.-Sitosterol & 0.00 & 0.00 & - & 1.89 \\
\hline 34.88 & Ergost-5-en-3-ol; (3.beta.)- & 0.77 & 1.04 & - & - \\
\hline 35.57 & Stigmasterol & 0.74 & 0.90 & - & - \\
\hline 37.33 & .gamma.-Sitosterol & 5.67 & 7.25 & - & - \\
\hline Total & & 10.75 & 12.60 & 9.13 & 18.94 \\
\hline \multicolumn{6}{|l|}{ Esters } \\
\hline 3.48 & Acetic acid; 2-methylpropyl ester & - & - & 0.42 & 5.20 \\
\hline 4.08 & Acetic acid; butyl ester & - & - & - & 0.87 \\
\hline 4.63 & 2-Pentanol; acetate & - & - & 0.77 & 1.26 \\
\hline 5.13 & 1-Butanol; 3-methyl-; acetate & - & - & 1.48 & 10.70 \\
\hline 6.51 & Butanoic acid; 2-methylpropyl ester & - & - & 0.36 & 0.91 \\
\hline 7.41 & Butanoic acid; 3-methyl-; 2-methylpropyl ester & - & - & - & 0.38 \\
\hline 7.50 & Propanoic acid; 2-methyl-; 3-methylbutyl ester & - & - & - & 0.63 \\
\hline 7.62 & 4-Hexen-1-ol; (4E)-; acetate & - & - & - & 0.28 \\
\hline 7.68 & Butanoic acid; 1-methylbutyl ester & - & - & 0.74 & 0.33 \\
\hline 7.80 & Propanoic acid; 2-methyl-; 1-methylethyl ester & - & - & 0.00 & 0.30 \\
\hline 8.25 & Butanoic acid; 3-methylbutyl ester & - & - & 2.61 & 3.44 \\
\hline 9.08 & Butanoic acid; 3-methyl-; 3-methylbutyl ester & - & - & 1.69 & 2.28 \\
\hline 10.72 & Butyric acid; 4-pentadecylester & - & - & 0.37 & - \\
\hline 19.51 & Hexadecanoic acid; methyl ester & 0.14 & 0.47 & - & - \\
\hline Total & & 0.14 & 0.47 & 8.45 & 26.60 \\
\hline \multicolumn{6}{|l|}{ Acids } \\
\hline 3.61 & Hexanoic acid & - & - & - & 1.43 \\
\hline 4.44 & Butanoic acid; 3-methyl- & 0.15 & 0.39 & 0.52 & 0.87 \\
\hline 6.85 & Hexanoic acid & 0.20 & 0.23 & - & - \\
\hline 10.06 & Octanoic acid & 0.53 & 0.00 & - & - \\
\hline 11.48 & 3-Nonenoic acid & 0.36 & - & - & - \\
\hline 14.54 & Azelaic acid & 8.55 & 0.50 & - & - \\
\hline 15.16 & 12-Hydroxydodecanoic acid & 0.92 & - & - & - \\
\hline 17.73 & Tetradecanoic acid & 0.42 & 0.45 & 0.35 & - \\
\hline 18.60 & (E)-13-Docosenoic acid & 0.24 & 4.07 & 0.59 & 0.53 \\
\hline 18.82 & Pentadecanoic acid & 0.70 & 0.81 & - & - \\
\hline 19.69 & cis-9-Hexadecenoic acid & 4.93 & 6.08 & 1.05 & 0.67 \\
\hline 19.98 & n-Hexadecanoic acid & 29.22 & 24.03 & 22.00 & 9.77 \\
\hline 20.64 & Oleic acid & 0.09 & 1.50 & 1.63 & - \\
\hline 20.84 & Heptadecanoic acid & 0.28 & 0.41 & - & - \\
\hline 21.52 & 9,12-Octadecadienoic acid (Z,Z)- & 0.25 & 10.56 & 1.73 & 0.85 \\
\hline 21.57 & 9,12,15-Octadecatrienoic acid; (Z,Z,Z)- & - & - & 5.94 & 2.87 \\
\hline
\end{tabular}


Table 1 (continued)

\begin{tabular}{|c|c|c|c|c|c|}
\hline \multirow[t]{2}{*}{ Retention time } & \multirow[t]{2}{*}{ Volatile compounds } & \multicolumn{4}{|c|}{ Relative content (\%) } \\
\hline & & $\mathrm{CK}$ & $\mathrm{T} 1$ & $\mathrm{~T} 2$ & $\mathrm{~T} 3$ \\
\hline 21.64 & cis-Vaccenic acid & 6.59 & - & - & - \\
\hline 21.68 & cis-10-Heptadecenoic acid & - & 10.00 & - & - \\
\hline 21.80 & Octadecanoic acid & 3.45 & 4.44 & 3.39 & 0.83 \\
\hline 23.70 & 17-Octadecynoic acid & 1.32 & - & - & - \\
\hline Total & & 58.20 & 63.46 & 37.22 & 17.82 \\
\hline \multicolumn{6}{|l|}{ Aldehyde } \\
\hline 3.88 & Hexanal & 0.65 & 1.33 & 0.83 & 1.98 \\
\hline 4.74 & 2-Hexenal; (E)- & 0.08 & 0.61 & 4.70 & 3.42 \\
\hline 5.34 & Hexanal; 3-methyl- & - & - & 0.57 & 0.31 \\
\hline 9.85 & 2,6-Nonadienal; (E,Z)- & 0.25 & - & - & - \\
\hline 9.96 & 2-Nonenal; (E)- & 0.22 & - & - & - \\
\hline 11.99 & 2,4-Decadienal & 0.38 & - & - & - \\
\hline 12.33 & 2,4-Decadienal; (E,E)- & 0.42 & - & - & - \\
\hline Total & & 2.01 & 1.94 & 6.10 & 5.71 \\
\hline \multicolumn{6}{|l|}{ Ketones } \\
\hline 2.48 & 2-Pentanone & - & - & 2.40 & 1.88 \\
\hline Total & & 0 & 0 & 2.40 & 1.88 \\
\hline \multicolumn{6}{|l|}{ Hydrocarbons } \\
\hline 2.35 & Pentane; 2,3-dimethyl- & 0.25 & 0.09 & - & 0.86 \\
\hline 2.64 & 2-Hexene; 4-methyl-; (E)- & 0.36 & 0.12 & - & - \\
\hline 10.58 & Dodecane & 2.14 & 2.86 & 6.09 & 0.41 \\
\hline 12.75 & Hexane; 3-ethyl-3-methyl- & 0.84 & - & - & - \\
\hline 13.32 & 1-Tridecene & 0.65 & 0.80 & 1.71 & 0.17 \\
\hline 13.43 & Tetradecane & 2.57 & 2.63 & 6.02 & 0.38 \\
\hline 15.60 & Hexadecane; 2,6,10,14-tetramethyl- & 0.16 & 0.21 & 0.35 & - \\
\hline 15.87 & 1-Pentadecene & 0.93 & 1.06 & 3.70 & - \\
\hline 15.96 & Hexadecane & 0.33 & 0.40 & 0.67 & - \\
\hline 17.12 & Eicosane & 0.17 & 0.19 & - & - \\
\hline 18.15 & 1-Octadecene & 0.84 & 1.01 & 2.16 & - \\
\hline 20.16 & 1-Nonadecene & 0.05 & 0.95 & 1.27 & - \\
\hline 23.03 & Pentacosane & 0.29 & - & - & - \\
\hline 23.88 & Tetracosane & 0.38 & 0.38 & - & - \\
\hline 27.45 & Hexatriacontane & 0.39 & 0.48 & - & - \\
\hline 27.63 & Squalene & 0.15 & 0.27 & - & - \\
\hline Total & & 10.50 & 11.47 & 21.97 & 1.82 \\
\hline \multicolumn{6}{|l|}{ Others } \\
\hline 2.20 & Butane; 1-ethoxy- & - & - & 1.83 & 7.71 \\
\hline 2.20 & Propane; 1-ethoxy-2-methyl- & 2.27 & 0.80 & - & - \\
\hline 3.28 & Pentane; 1-ethoxy- & 5.82 & 3.16 & 5.03 & 14.82 \\
\hline 3.43 & Toluene & 0.90 & 0.39 & 1.85 & 1.87 \\
\hline 4.90 & Ethylbenzene & 0.44 & 0.33 & 0.85 & 0.78 \\
\hline 14.31 & 2,5-di-tert-Butyl-1,4-benzoquinone & 0.22 & 0.23 & 0.36 & 0.00 \\
\hline 15.27 & Benzene; 1,2,3-trimethoxy-5-(2-propenyl)- & - & - & 0.95 & 0.47 \\
\hline 16.59 & 2-n-Octylfuran & 0.11 & 1.10 & 0.49 & - \\
\hline 19.36 & 7,9-Di-tert-butyl-1-oxaspiro(4,5)deca-6,9-die & 0.25 & 0.29 & - & - \\
\hline 19.85 & Dibutyl phthalate & 3.42 & - & - & - \\
\hline 21.97 & Hexadecanamide & 0.72 & 0.46 & 0.60 & 0.23 \\
\hline 23.56 & 9-Octadecenamide; (Z)- & 1.59 & 1.63 & 1.56 & 0.75 \\
\hline 23.77 & Octadecanamide & 0.47 & 0.29 & 0.42 & 0.18 \\
\hline
\end{tabular}


Table 1 (continued)

\begin{tabular}{llllll}
\hline Retention time & Volatile compounds & \multicolumn{4}{l}{ Relative content (\%) } \\
\cline { 3 - 6 } & & $\mathrm{CK}$ & $\mathrm{T} 1$ & $\mathrm{~T} 2$ & $\mathrm{~T} 3$ \\
\hline 23.99 & Phenol; 2,2'-methylenebis[6-(1,1-dimethylethy & 0.45 & 0.39 & - & - \\
27.23 & 13-Docosenamide; (Z)- & 1.74 & 0.98 & 0.79 & 0.42 \\
Total & & 18.39 & 10.05 & 14.73 & 27.23 \\
\hline
\end{tabular}



Fig. 2 DEGs identified among four ripening stages of M. acuminata fruits: "filled triangle" refers to upregulation and "inverted filled triangle" refers to downregulation

from CK during T1, T2, and T3 periods; complete details are given in File S1.

\section{KEGG Functional Enrichment Analysis of DEGs}

The most crucial aroma compounds include, among others, amino acid-derived compounds, lipid-derived compounds, phenolic derivatives, and mono- and sesquiterpenes (Schwab et al. 2008). In this study, through the KEGG enrichment analysis of 9630 genes that were differentially expressed in the T1, T2, and T3 periods with CK, it was determined that when $P \leq 0.05$. The identified DEGs in profile 21 were annotated with 21 biological processes, 13 cellular components and 12 molecular functions in GO categories (Figure S1). Among the biological processes, a great number of DEGs were focused on categories of "metabolic process", "cellular process", "single-organism process", "biological regulation", "regulation of biological process", "localization", "response to stimulus" and "cellular component organization or biogenesis"; The terms of "cell", "cell part", "organelle", "macromolecular complex", "organelle part", "membrane" and " membrane part" were the dominant groups in the cellular component; "binding", "catalytic activity" and "transporter activity" were the representative groups in the molecular function. Furthermore, the significantly DEGs were blasted to the KEGG database to analyze their biological pathways. Among 111 of the enriched KEGG pathways, the most significant ones $(Q \leq 0.05)$ consist of "Oxidative phosphorylation", "Glycolysis/Gluconeogenesis", "Pyruvate metabolism", "Carbon fixation in photosynthetic organisms" and "Citrate cycle (TCA cycle)" (Figure S2), followed by others, such as "Valine, leucine and isoleucine degradation", "Propanoate metabolism", "SNARE interactions in vesicular transport", "Pantothenate and CoA biosynthesis", and "Glucosinolate biosynthesis".

The linoleic acid metabolism pathway was not only enriched in the top 20, but was significantly enriched as well (Fig. 3a). By extracting the genes upregulated with the CK stage, and then doing KEGG enrichment analysis, when $P \leq 0.05$, it was found that among the top 20 pathways of enrichment degree, the enrichment of valine, leucine, and isoleucine degradation pathway obtained a place in the top 20 and proved to be significantly enriched (Fig. 3b). Also, these two pathways are the main pathways of aroma synthesis (Yvon and Rijnen 2001; Dudareva et al. 2013). The above research indicates that the formation of aroma substances during the post-harvesting process of bananas could establish the possible relationship of this formation to the two metabolic pathways of linoleic acid metabolism and valine, leucine, and isoleucine degradation.

\section{DEGs Related to Valine, Leucine, and Isoleucine Degradation and Linoleic Acid Metabolism}

The LOX metabolic pathway is mainly responsible for the degradation of unsaturated fatty acids, one of the most prominent examples being (E)-2-hexenal with its intense fresh green, green apple-like sensory properties that acts as a key enhancer of the flavor of many fruits and vegetables (Dudareva et al. 2013). Investigations suggest that at least four enzymes are involved in the biosynthetic pathway leading to their formation: lipoxygenase (LOX), hydroperoxide lyase (HPL), 3Z,2E-enal isomerase, and alcohol dehydrogenase (ADH).

In the linoleic acid degradation pathway, the expression levels of LOX gene and ADH gene were found to be active in $\mathrm{T} 2$ and $\mathrm{T} 3$ stages, which can presumably be related to the increase in the content of Hexanal and 2-Hexenal; (E)- at the ripening stage of banana fruits. Dulermo et al. suggested that TGL genes are key performers in aroma synthesis (Dulermo 
$\mathbf{a}$



Fig. 3 Top 20 significantly enriched KEGG pathway of DEGs. a KEGG enrichment analysis results of 9630 genes that were differentially expressed with CK at T1, T2, and T3. b Extracted upregulated genes compared with the CK stage for KEGG enrichment analysis results. The $Y$ axis on the left represents KEGG pathways, and the

et al. 2013). In this study, heat maps of genes enriched in linoleic acid metabolism revealed that 15 genes were enriched in this pathway. Further analysis revealed that MaTGL4 (GSMUA-Achr3G14540-001) was directly involved in the linoleic acid metabolism pathway, and the expression of MaTGL4 was significant at the later stage of ripening (Fig. 4), pointing out clearly that MaTGL4 gene may also be involved in regulating the synthesis of banana aroma.

The direct metabolic pathway of amino acids leads to the formation of aliphatic, branched, and aromatic alcohols, aldehydes, and ketones as well as acids and esters (Dudareva et al. 2013). The major aroma compounds derived from branched-chain and aromatic amino acids and methionine, valine, leucine, and isoleucine are the main branched-chain amino acids (Yvon and Rijnen 2001). When leucine is the initial free amino acid, 3-methylbutanol and 3-methylbutanoic acid are formed, whereas isoleucine would be the precursor for 2-methylbutanol and 2-methylbutanoic acid. The branched acids may further esterify with alcohols to form the corresponding esters (Yvon and Rijnen 2001). In this paper, a heat map of genes enriched in the valine, leucine, and isoleucine degradation pathways was performed, where it was found that MaBCAT3 (GSMUA-Achr3G15170-001) and MaBCAT5 (GSMUA-Achr3G15180-001) are directly involved in the valine, leucine, and isoleucine degradation b

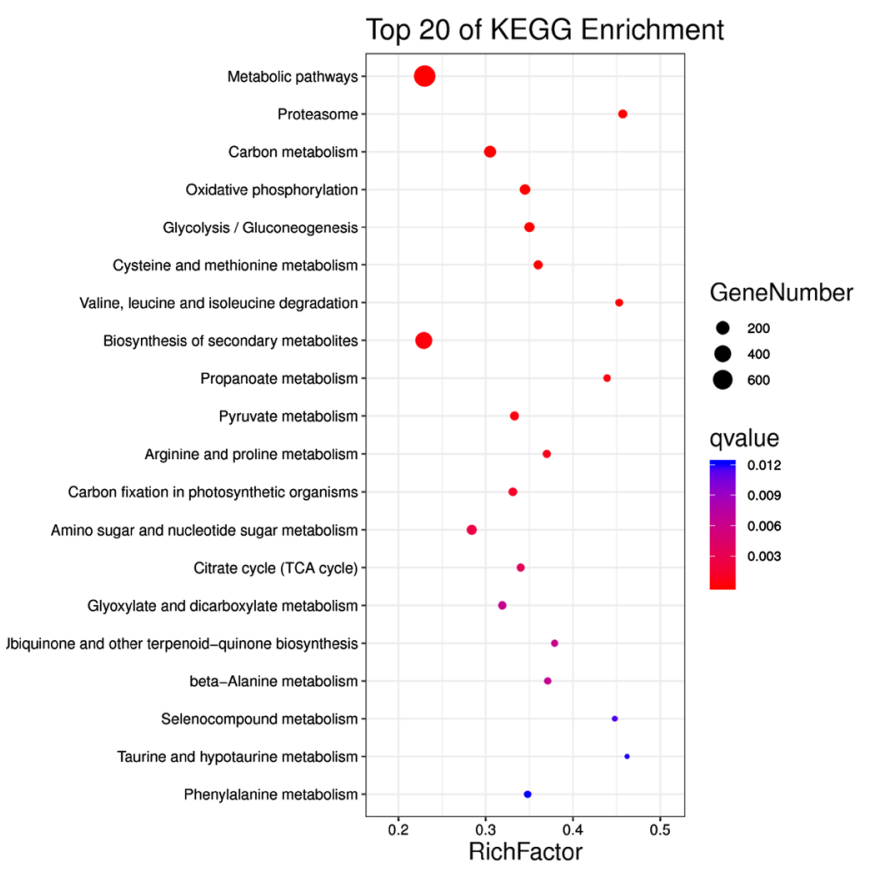

$X$ axis indicates the "enrich factor" represented by the ratio of number of DEGs to the number of total annotated genes of each pathway. Low $q$-values are shown in the red circle, and high $q$-values are shown in the green circle. The area of a circle represents the number of DEGs

pathways, and they all belong to the branched-chain amino acid aminotransferase (BCAT) gene family. Guo et al. showed that branched-chain amino acid transaminase 1 (BCAT1) gene is related to the synthesis of aromatic substances (Miranda et al. 2001). The development of a pleasant aroma during fruit ripening is a trait of the metabolism of branched amino acids such as leucine with deamination and decarboxylation (Dou et al. 2020). The expression of $M a B C A T 3$ and MaBCAT5 genes screened in this study were found to be active in the late ripening stage in the banana fruit (Fig. 5), indicating that these two genes may dominantly regulate the formation of aroma substances in the late ripening stage of bananas.

Volatile metabolites, such as aliphatic saturated or unsaturated alcohols, aldehydes, ketones, acids, esters, or lactones, derived from fatty acids as non-volatile precursors remain a crucial parameter for the flavor of most fruits and vegetables. $\beta$-Oxidation is believed to be the main pathway for the degradation of straight-chain fatty acids in intact fruits and vegetables (Goepfert and Poirier 2007). In $\beta$-oxidation, the acyl-CoA derivatives of fatty acids are degraded stepwise so that in every reaction cycle the chain is shortened by two $\mathrm{C}$ atoms. Finally, acetic acid or butyric acid is generated, and the main aroma is catalyzed by AAT 
LINOLEIC ACID METABOLISM

a
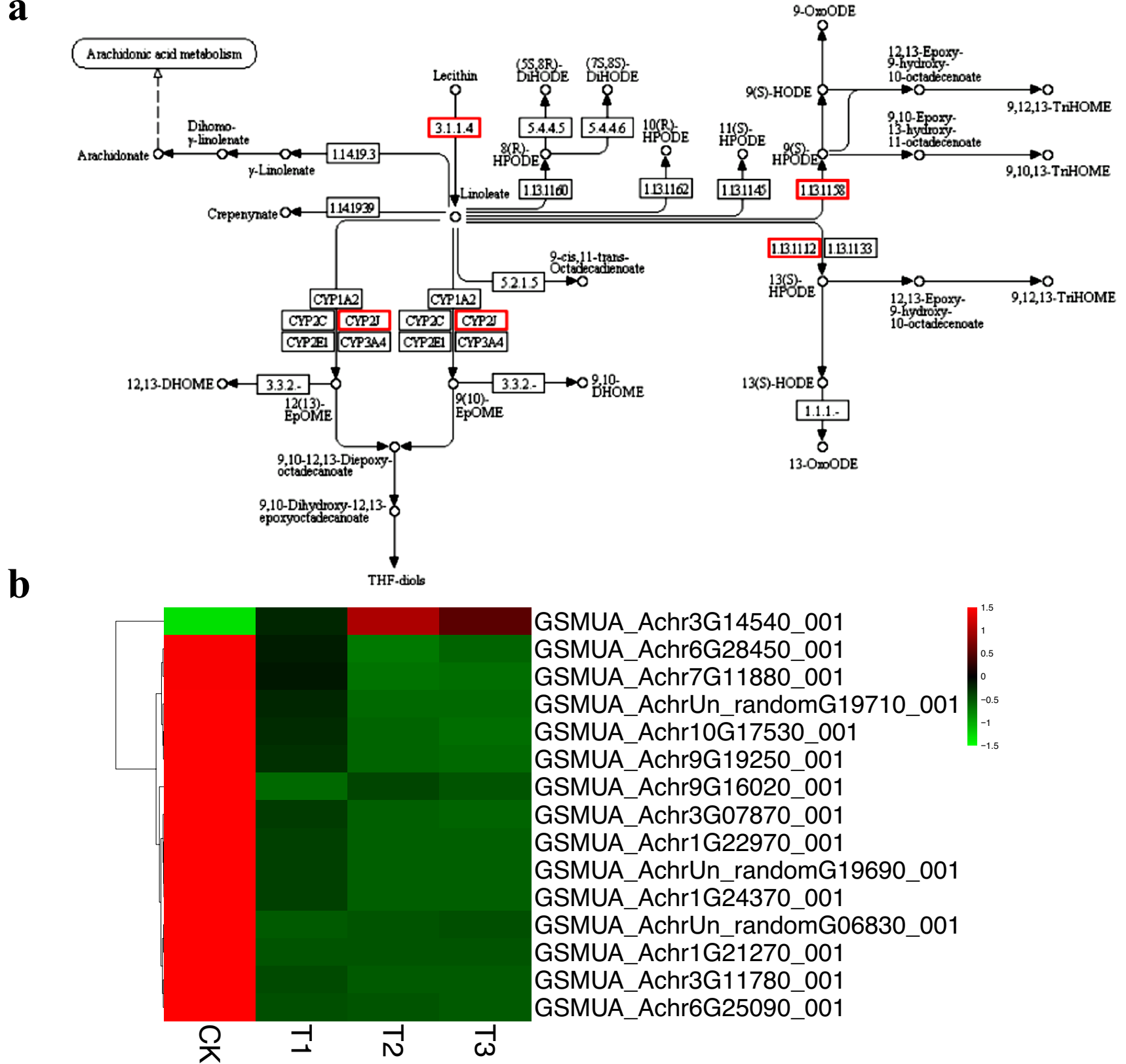

Fig. 4 Gene heat map enriched in the linoleic acid metabolism pathway. a Linoleic acid metabolism pathway diagram. b Genes enriched in the linoleic acid metabolism pathway are expressed at different post-harvesting stages

to produce branched-chain acetate or butyrate (Baker et al. 2006; Bruckner and Wyllie 2008). Our transcriptome results show that during fatty acid degradation pathway, the expression level of genes involved in $\beta$-oxidation in T1 or T2 were significantly elevated. The upregulation of these genes may be related to the degradation of aroma component n-Hexadecanoic acid and the formation of branched acetate or butyrate.

\section{qRT-PCR Validation}

To validate the reliability of genes experimentally (obtained from the assembled transcriptome of gene expression using RNA-Seq data), a total of 8 genes were selected for qRTPCR. As shown in Fig. 6, all the expression patterns of these genes, as detected by qRT-PCR, were essentially consistent with those obtained from RNA-Seq data, which established 
$\mathbf{a}$

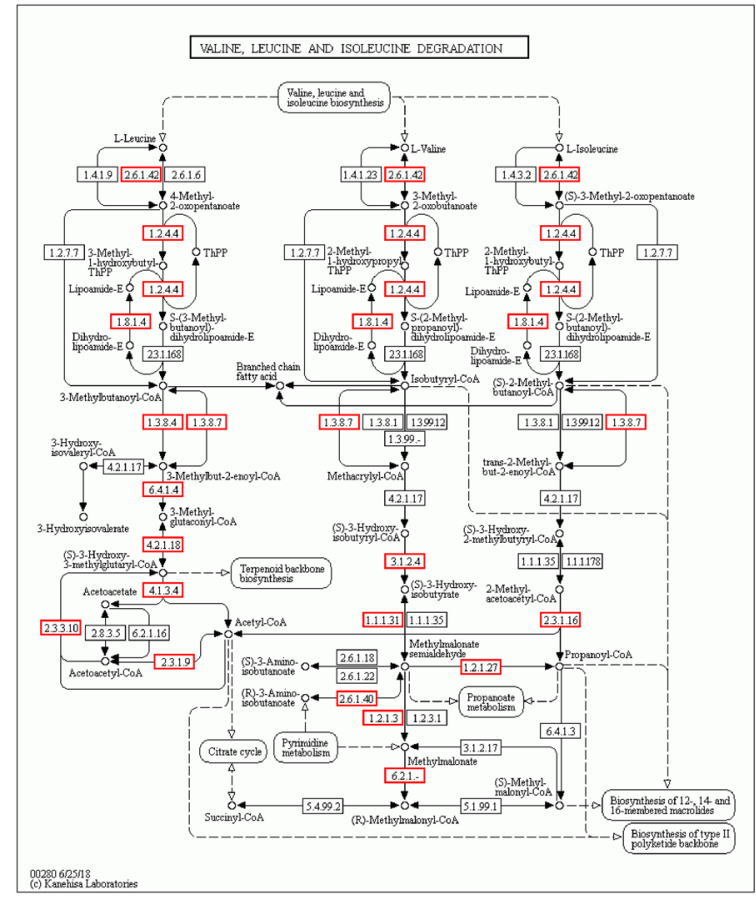

b

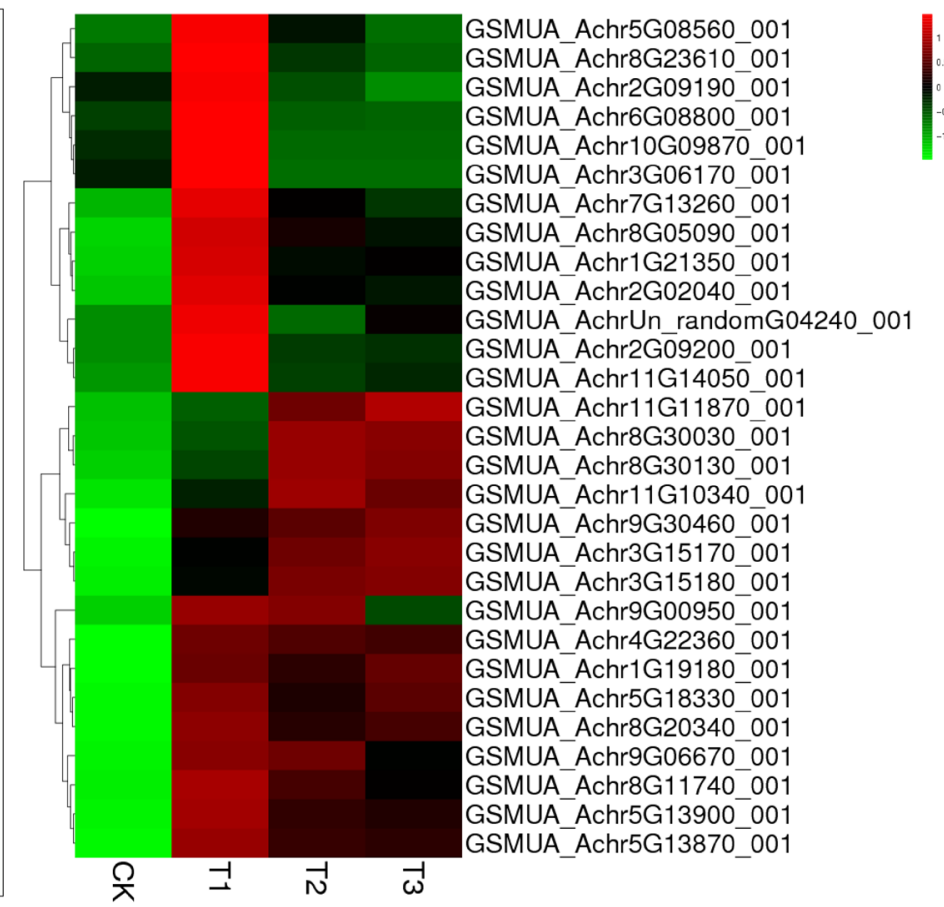

Fig. 5 Gene heat map enriched in the valine, leucine, and isoleucine degradation pathway. a Valine, leucine, and isoleucine degradation pathway map. b Genes enriched in the valine, leucine, and isoleucine degradation pathway are expressed at different post-harvesting stages

that the genes obtained from the assembled transcriptome are valid, and gene expression profiles from RNA-Seq data are reliable. Noticeably, the expression of the two genes of the valine, leucine, and isoleucine biosynthesis pathways, MaBCAT3 (GSMUA-Achr3G15170-001) and MaBCAT5 (GSMUA-Achr3G15180-001), with the extension of the post-harvesting stage, were significantly increased, and the trend is consistent with transcription group (File S2). This demonstrates that these two genes may dominantly regulate the synthesis of aroma substances in the late ripening period of bananas. In the linoleic acid metabolism pathway, the MaTGL4 (GSMUA-Achr3G14540-001) gene proved to have a tendency to first increase and then decline with the extension of the post-harvesting stage, which is consistent with the transcriptome trend (File S2). The reason could be the early appearance of the gene expression. The aroma substance is released for a period of time after the expression level rises, and the T3 phase of the post-harvesting of the banana seems to be the final step of the post-harvesting process of the banana, until aroma substances accumulate to a certain degree, and gene expression is not significantly expressed relative to $\mathrm{T} 2$, but is maintained at a certain expression level, or even diminishes further.

\section{Conclusions}

Bananas are picked during the post-harvesting stage and they enter the edible stage after the post-harvesting process is completed. The physiological and biochemical indicators of banana fruits show tremendous variations at different post-harvesting stages, such as solid color, fruit hardness, fruit quality, fruit aroma. Among them, the change of VOCs in the post-harvesting process serves as one of the most vital indicators that dominantly affect the flavor of bananas. Therefore, it is of great theoretical and practical significance to study the changing rules and mechanisms of banana VOCs in different post-harvesting stages. In this study, firstly, the GC-MS technology was utilized to detect the changes of VOCs in various post-harvesting stages of bananas, and then was further combined with transcriptomics technology to discover three candidate genes involved in the synthesis of VOCs in different post-harvesting stages of bananas. The data deduced by this study not only provides a better understanding of the mechanism of banana VOC synthesis in different post-harvesting stages, but also lays out a basic guide to the design of sophisticated strategies to enhance 
a

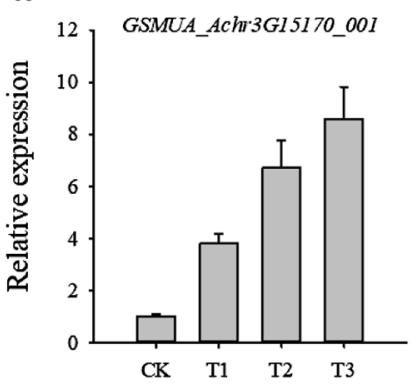

e

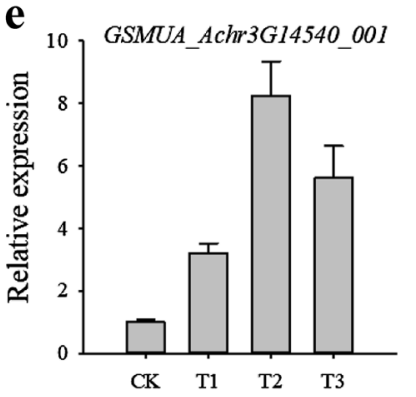

b

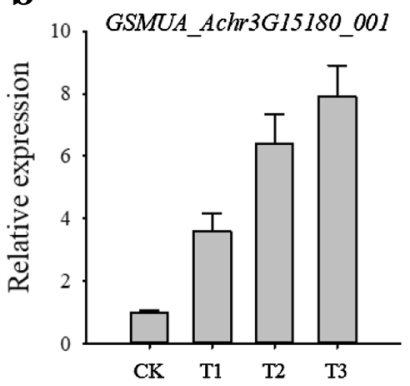

f

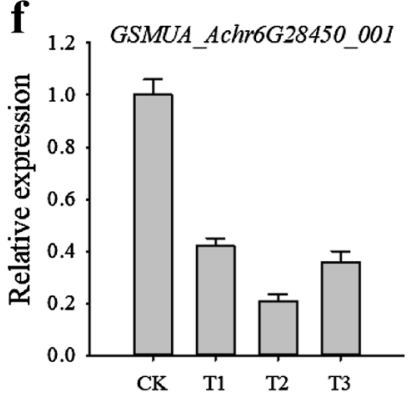

c
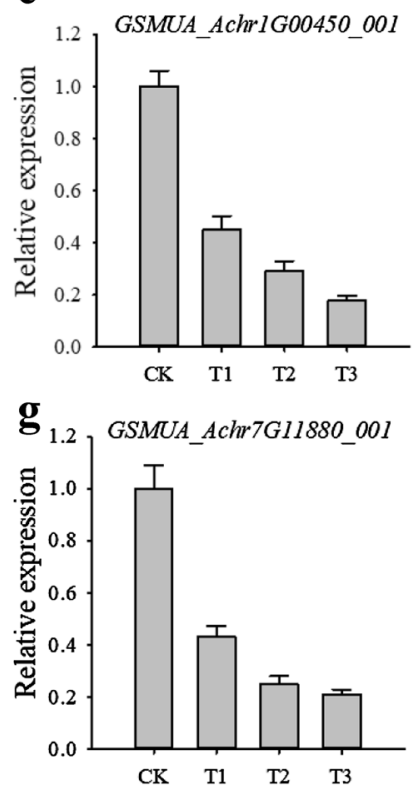

d

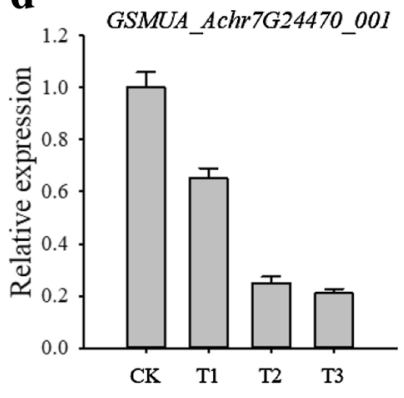

$\mathbf{h}_{1.2}$ GSMUA_Achr3G07870_001

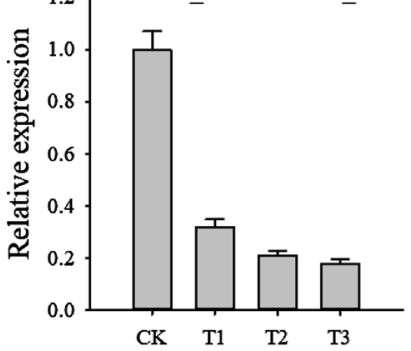

Fig. 6 qRT-PCR analysis of aroma components biosynthesis genes during the different post-harvesting stages of cavendish banana fruit

banana post-harvesting aroma components through genetic improvement.

Supplementary Information The online version contains supplementary material available at https://doi.org/10.1007/s00344-021-10496-y.

Acknowledgements This work was supported by the National Key R\&D Program of China Nos. 2018YFD1000200 and 2019YFD1000900; Science and Technology Plan Project of Guangzhou City No. 201903010010; Modern Agricultural Innovation Team Project of Guangdong Province No. 2019KJ109; The Natural Science Foundation of Guangdong Province, No. 2019A1515012120; National Natural Science Foundation of China, No. 31801843; Guangdong Academy of Agricultural Sciences Foundation, No. 201815; Tutor system project of Guangdong Academy of Agricultural Sciences, No. R2018QD-018.

Author Contributions Conceptualization, TD; Methodology, $\mathrm{CH}$ and SZ; Software, YL; Validation, HG and WH; Formal analysis, GD; Investigation, OS and FB; Resources, QY; Data curation, CL; Writing — original draft preparation, TD; Writing — review and editing, GY and TD; Visualization, FB; Supervision, TD and GY; Project administration, FB, TD, and CL; Funding acquisition, TD and GY. All authors have read and agreed to the published version of the manuscript.

\section{Declarations}

Conflict of interest The authors declare no conflict of interest. The funders had no role in the design of the study; in the collection, analyses, or interpretation of data; in the writing of the manuscript, or in the decision to publish the results.
Open Access This article is licensed under a Creative Commons Attribution 4.0 International License, which permits use, sharing, adaptation, distribution and reproduction in any medium or format, as long as you give appropriate credit to the original author(s) and the source, provide a link to the Creative Commons licence, and indicate if changes were made. The images or other third party material in this article are included in the article's Creative Commons licence, unless indicated otherwise in a credit line to the material. If material is not included in the article's Creative Commons licence and your intended use is not permitted by statutory regulation or exceeds the permitted use, you will need to obtain permission directly from the copyright holder. To view a copy of this licence, visit http://creativecommons.org/licenses/by/4.0/.

\section{References}

Ardö Y (2005) Flavour formation by amino acid catabolism. Biotechnol Adv 24(2):238-242

Asif MH, Lakhwani D, Pathak S, Gupta P, Bag SK, Nath P, Trivedi PK (2014) Transcriptome analysis of ripe and unripe fruit tissue of banana identifies major metabolic networks involved in fruit ripening process. BMC Plant Biol 14(1):316

Aurore G, Ginies C, Ganou-Parfait B, Renard CMGC, Fahrasmane L (2011) Comparative study of free and glycoconjugated volatile compounds of three banana cultivars from french west indies: cavendish, frayssinette and plantain. Food Chem 129(1):28-34

Baker A, Graham IA, Holdsworth M, Smith SM, Theodoulou FL (2006) Chewing the fat: $\beta$-oxidation in signalling and development. Trends Plant Sci 11:124-132

Beekwilder J, Alvarez-Huerta M, Neef E, Verstappen FWA, Bouwmeester HJ, Aharoni A (2004) Functional characterization of 
enzymes forming volatile esters from strawberry and banana. Plant Physiol 135:1865-1878

Boudhrioua N, Giampaoli P, Bonazzi C (2003) Changes in aromatic components of banana during ripening and air-drying. LWT Food Sci Technol 36(6):633-642

Brat P, Yahia A, Chillet M, Bugaud C, Bakry F, Reynes M, Brillouet JM (2004) Influence of cultivar, growth altitude and maturity stage on banana volatile compound composition. Fruits 59(2):75-82

Bruckner B, Wyllie SG (2008) Fruit and vegetable flavour: recent advances and future prospects. Woodhead Publishing, Cambridge, pp 41-70

Bugaud C, Alter P (2016) Volatile and non-volatile compounds as odour and aroma predictors in dessert banana (Musa spp.). Postharvest Biol Technol 112:14-23

Chen Q, Song J, Bi J, Meng X, Wu X (2018) Characterization of volatile profile from ten different varieties of Chinese jujubes by HSSPME/GC-MS coupled with E-nose. Food Res Int 105:605-615

Dong T, Chen XJ, Wang M, Huang YH, Yi GJ (2014) Comparison of volatile aroma compounds in Dwarf Cavendish banana (Musa spp. AAA) grown under organic or traditional cultivation. J Hortic Sci Biotechnol 89(4):441-447

Dou TX, Hu CH, Sun XX, Shao XH, Wu JH, Ding LJ et al (2016) MpMYBS3 as a crucial transcription factor of cold signaling confers the cold tolerance of banana. Plant Cell Tiss Organ Cult 125:93-106

Dou TX, Shi JF, Li Y, Bi FC, Gao HJ, Hu CH et al (2020) Influence of harvest season on volatile aroma constituents of two banana cultivars by electronic nose and HS-SPME coupled with GC-MS. Hortic Sci. https://doi.org/10.1016/j.scienta.2020.109214

Dudareva N, Klempien A, Muhlemann JK, Kaplan I (2013) Biosynthesis, function and metabolic engineering of plant volatile organic compounds. New Phytol 198:16-32

Dulermo T, Tréton B, Beopoulos A, Kabran GAP, Haddouche R, Nicaud JM (2013) Characterization of the two intracellular lipases of $\gamma$. lipolytica encoded by TGL3 and TGL4 genes: new insights into the role of intracellular lipases and lipid body organisation. Biochim Biophys Acta 1831(9):1486-1495

Facundo HVDV, Garruti DDS, Dias CTDS, Cordenunsi BR, Lajolo FM (2012) Influence of different banana cultivars on volatile compounds during ripening in cold storage. Food Res Int 49:626-633

Frérot B, Leppik E, Groot AT, Unbehend M, Holopainen JK (2017) Chemical signatures in plant: insect interactions. Adv Bot Res 81:139-177

Goepfert S, Poirier Y (2007) $\beta$-Oxidation in fatty acid degradation and beyond. Curr Opin Plant Biol 10(3):245-251

Guo YF, Zhang YL, Shan W, Cai YJ, Liang SM, Chen JY et al (2018) Identification of two transcriptional activators MabZIP4/5 in controlling aroma biosynthetic genes during banana ripening. J Agric Food Chem 66(24):6142-6150

Hadi E, Mohamed MA, Zhang FJ, Wu FF, Zhou CH, Tao J (2013) Advances in fruit aroma volatile research. Molecules 18(7):8200-8229

Huang C, Zhao FG, Lin Y, Zheng SP, Liang SL, Han SY (2018) RNASeq analysis of global transcriptomic changes suggests a roles for the MAPK pathway and carbon metabolism in cell wall maintenance in a Saccharomyces cerevisiae FKS1 mutant. Biochem Biophys Res Commun 500(3):603-608

Itay G, Einat B, Vitaly P, Shery L, Joseph B, Arthur SA et al (2010) Branched-chain and aromatic amino acid catabolism into aroma volatiles in Cucumis melo L. fruit. J Exp Bot 61(4):1111-1123

Jordan MJ, Tandon K, Shaw PE, Goodner KL (2001) Aromatic profile of aqueous banana essence and banana fruit by gas chromatography-mass spectrometry (GC-MS) and gas chromatography-olfactometry (GC-O). J Agric Food Chem 49(10):4813-4817
Li B, Dewey CN (2011) RSEM: accurate transcript quantification from RNA-Seq data with or without a reference genome. BMC Bioinform 12:323

Li B, Fan RY, Yang QS, Hu CH, Sheng O, Deng GM et al (2020) Genome-wide identification and characterization of the NAC transcription factor family in Musa acuminata and expression analysis during fruit ripening. Int J Mol Sci 21(2):634

Marriott J, Palmer JK (1980) Bananas-physiology and biochemistry of storage and ripening for optimum quality. Crit Rev Food Sci Nutr 13(1):41-88

Mayr D, Märk T, Lindinger W, Brevard H, Yeretzian C (2003) Breathby-breath analysis of banana aroma by proton transfer reaction mass spectrometry. Int J Mass Spectrom 223-224:743-756

Miranda EJF, Nogueira RI, Sérgio MP, Rezende CM (2001) Odouractive compounds of banana passa identified by aroma extract dilution analysis. Flavour Frag J 16(4):281-285

Nogueira JMF, Fernandes PJP, Nascimento AMD (2003) Composition of volatiles of banana cultivars from Madeira Island. Phytochem Anal 14(2):87-90

Pérez AG, Raqyel O, Luaces P, Sanz C (2002) Biosynthesis of strawberry aroma compounds through amino acid metabolism. J Agric Food Chem 50(14):4037-4042

Pino JA, Febles Y (2013) Odour-active compounds in banana fruit cv. Giant Cavendish. Food Chem 141(2):795-801

Pino JA, Winterhalter P, Benitez MC (2017) Odour-active compounds in baby banana fruit (Musa acuminata AA Simmonds cv. Bocadillo). Int J Food Prop 20(5):1448-1455

Pontes M, Pereira J, Camara JS (2012) Dynamic headspace solidphase microextraction combined with one-dimensional gas chromatography-mass spectrometry as a powerful tool to differentiate banana cultivars based on their volatile metabolite profile. Food Chem 134(4):2509-2520

Rustagi A, Jain S, Kumar D, Shekhar S, Jain M, Bhat V, Sarin NB (2015) High efficiency transformation of banana [Musa acuminate L. cv. Matti (AA)] for enhanced tolerance to salt and drought stress through overexpression of a peanut salinityinduced pathogenesis-related class 10 protein. Mol Biotechnol 57(1):27-35

Saha B, Bucknall MP, Arcot J, Driscoll R (2018) Profile changes in banana flavour volatiles during low temperature drying. Food Res Int 106:992-998

Santos TLRD, Ortiz MMM, Tatiane MR (2017) Herbivory by leaf-cutter ants changes the glandular trichomes density and the volatile components in an aromatic plant model. AoB Plants 9(6):plx057

Schwab W, Davidovich-Rikanati R, Lewinsohn E (2008) Biosynthesis of plant-derived flavor compounds. Plant J 54(4):712-732

Selli S, Gubbuk H, Kafkas E, Gunes E (2012) Comparison of aroma compounds in Dwarf Cavendish banana (Musa spp. AAA) grown from open-field and protected cultivation area. Hortic Sci 141:76-82

Shivashankara K, Pavithra K, Geetha G, Roy T, Patil P, Menon R (2017) Seasonal influence on volatile aroma constituents of two banana cultivars (Grand Naine and Nendran) under Kerala conditions. Hortic Sci 12:124-132

Souleyre EJF, Chagné D, Chen XY, Tomes S, Turner RM, Wang MY et al (2014) The AAT1 locus is critical for the biosynthesis of esters contributing to "ripe apple" flavour in "Royal Gala" and "Granny Smith" apples. Plant J 78(6):903-915

Speirs J, Lee E, Holt K, Yong-Duk K, Scott NS, Loveys B, Schuch W (1998) Genetic manipulation of alcohol dehydrogenase levels in ripening tomato fruit affects the balance of some flavor aldehydes and alcohols. Plant Physiol 117(3):1047-1058

Wang J, Li Y, Chen R, Bao J, Yang G (2007) Comparison of volatiles of banana powder dehydrated by vacuum belt drying, freeze-drying and air-drying. Food Chem 104(4):1516-1521 
Wei Y, Hu W, Wang Q, Zeng H, Li X, Yan Y et al (2017) Identification, transcriptional and functional analysis of heat-shock protein 90s in banana (Musa acuminata L.) highlight their novel role in melatonin-mediated plant response to Fusarium wilt. J Pineal Res 62(1):e12367

Wyllie SG, Fellman JK (2000) Formation of volatile branched chain esters in bananas (Musa sapientum L.). J Agric Food Chem 48:3493-3496

Xu K, Wang J, Wei Z, Deng F, Wang Y, Cheng S (2017) An optimization of the MOS electronic nose sensor array for the detection of Chinese pecan quality. J Food Eng 203(JUN):25-31

Yang XT, Song J, Fillmore S, Pang XQ, Zhang ZQ (2011) Effect of high temperature on color, chlorophyll fuorescence and volatile biosynthesis in green-ripe banana fruit. Postharvest Biol Technol 62:246-257

Yvon M, Rijnen L (2001) Cheese flavour formation by amino acid catabolism. Int Dairy J 11:185-201

Zhu H, Li XP, Yuan RC, Chen YF, Chen WX (2010) Changes in volatile compounds and associated relationships with other ripening events in banana fruit. J Hort Sci Biotechnol 85(4):283-288

Zhu X, Li Q, Li J, Luo J, Chen W, Li X (2018) Comparative study of volatile compounds in the fruit of two banana cultivars at different ripening stages. Molecules 23:e2456

Publisher's Note Springer Nature remains neutral with regard to jurisdictional claims in published maps and institutional affiliations. 